\title{
Nanoanesthesia - A Novel Approach to Local Anesthesia Drug Delivery
}

\author{
Vijitha B* \\ Department of Cardiac Anesthesia, Jayadeva Institute of Cardiovascular Sciences \& \\ Research, India \\ *Corresponding author: Burra Vijitha, DM Resident, Department of Cardiac Anesthesia, \\ Jayadeva Institute of Cardiovascular Sciences \& Research, Bangalore, India, Email: \\ burravijitha@gmail.com
}

\section{Mini Review \\ Volume 3 Issue 3}

Received Date: June 26, 2018

Published Date: July 05, 2018

DOI: $10.23880 /$ nnoa- 16000142

\section{Abstract}

A technique using anesthesia containing nanoparticles, drawn to the targeted areaof body by magnets provide a useful alternative to nerve block for local anesthesia. This technique in patients suggests an experimental study. The engineered nanoparticle complexes which contain small amounts of Ropivacaine and the iron oxide mineral magnetite. Magnet derived nanoparticles-ropivacaine complexes are injected into the veins and then magnets placed around ankle for 15, 30, $60 \mathrm{~min}$. The goal was to use magnets to draw nanoparticles to ankleand particles release the anesthetic, numbing the nerves around the ankle. So, it isan alternative to local anesthetic block like that used for foot and ankle surgeriesand deliver high concentrations of local anesthetic directly to desired area withoutincreasing toxic effects. By this, it allows more potent doses of local anesthesia tobe delivered safely during local anesthesia in humans. By the disposition ofcomplexes at ankle with each cardiac cycle, are sequestered by liver, spleen, other organs and by magnet. With this technique, a safe dose of ropivacaine when combined with MNPs is higher when injected by iv.

Keywords: Nanoparticles; Ropivacaine; Magnet Derived Nanoparticles; Local Anaesthesia

\section{Introduction}

The concept of nanotechnology was first brought by Nobel Laureate Richard Feynman in 1959. Prof. Norio Taniguchi and Dr. Eric Dressler in 1970s and 1980s, respectively, had contributions in the initial propagation of the concept. It involves structures of one to several hundred nanometres in size. With nanotechnology, a specific set of materials and improved products can be designed by some changes in the microphysical structure. The biological and medical research communities have exploited the unique properties of nanomaterials for various applications. Functionalities can be added to nanomaterials by interfacing them with biological molecules [1]. The size of nanomaterials is similar to that of most biological structures, therefore useful for both in vivo and in vitro applications.

Nanotechnology has been a boon by delivering drugs to specific cells using nanoparticles. The principle exploited is overall drug consumption and side effects can be lowered by depositing the active agent only in the 


\section{Nanomedicine \& Nanotechnology Open Access}

morbid region and in no higher dosage than needed. This highly selective approach reduces the side effects and cost, by targeting its goal efficiently. Another principle which uses nanotechnology is the use of block copolymers, which form micelles for drug encapsulation. They hold small drug molecules, transporting them to the desired location. Also, improved emulsion delivery systems based on nanotechnology directly transport the molecule to end receptors. Nanotechnology also has new modalities in implantable drug delivery systems. These implantables are advantageous over injectables because they do not follow first-order kinetics as injectables [2]. Drugs displaying first-order kinetics have a rapid rise in their concentration and an exponential drop.This rapid rise can cause difficulties with toxicity and side effects, and drug efficacy can diminish as the drug concentration falls below the targeted range.

Bupivacaine overdose which anaesthesiologists encounter complications due to local anaesthetic overdose such as high spinal. At present, there are no antidotes and one has to wait for the drug to metabolise. With nanotechnology, an antidote to bupivacaine overdose is possible. There is a formation of pi-pi complexes between bupivacaine and a pi-electron-rich injectable nanoparticle. This complex is devoid of the clinical effects of bupivacaine and would thus render toxic bupivacaine harmless.

Pain and palliative care is the upcoming super speciality of anaesthesiology where narcotics are used for managing chronic pain. But these have systemic effects and have significant addiction potential. To overcome this, sustained release anaesthetics have been tried but unsuccessfully as the anaesthetics and their preservatives caused local toxicity. The solution to this is reflected in the use of saxitoxin, a potent anaesthetic, bundled with liposomes. This slow- release formulation can produce a nerve block lasting from days to weeks and even months, at the same time being nontoxic to the nerves or the surrounding tissue. This formulation possible with nanotechnology can potentially revolutionise the treatment of chronic and even acute pain.

\section{Few Future Concepts are Vasculoids, Respirocytes and Clottocytes}

Respirocytes: These nanostructures transport oxygen in human body similar to erythrocytes. Originally proposed by Robert Frietas in 1996, they are less than a micron in diameter, possibly made of diamond, a biocompatible material [3]. They also transport carbon dioxide. The proposed structure consists of three chambers. One would store oxygen, the other carbon dioxide, and the third would act as a buoyancy chamber, making the structure floatable in blood. The structure would also have rotors to control the intake and exit of carbon dioxide and oxygen, and for a controlled entry of glucose inside the structure to combine with oxygen and produce energy for the activity of respirocyte.

A popular dictum in science was that no machine is more efficient than the human body, but this invention would change this as it is capable of releasing cent percent of its oxygen store, whereas a natural erythrocyte is estimated to be capable of releasing only about onefourth of the stored gas. Even a modification of these respirocytes is on a design proposal that can quickly remove certain poisonous substances from the body in poisoning patients, for example, CO poisoning, nitrobenzene poisoning, etc. Respirocytes would also speed up weaning from ventilators. At present, prolonged ventilator stay is a demon for the intensivist as it makes the patient prone to Ventilator Associated Pneumonia (VAP). VAP decreases the oxygen diffusion, making a patient further dependent on the ventilator. Once respirocytes are practically successful, they can bypass this ventilator dependency and would prove to be a pillar of strength to the intensivist.

Biomedical and biotechnological applications of magnetic nanoparticles and their dispersions in liquids are found to be potentially useful [4]. Experimentally, ankle block in the rat done by IV injection of MNP \{Magnetic Nano Particles\}/Ropivacaine complexes with magnet application at the ankle. Possible disposition of the complexes at the ankle could be that with each cardiac cycle, they are sequestered by the liver, spleen, other organs, and by the magnet [5]. In the next several minutes, the complexes permeate the capillaries and accumulate in the ankle tissue. Irreversible ropivacaine release occurs; only the free drug is expected to be active. The released drug is no longer under the magnet's influence; some of it acts on the nerves with which it comes in contact, and some gets reabsorbed into the circulation.

Thirty minutes of magnet application to the ankle of animals injected with MNP/Ropiv was more effective than 15 or 60 minutes of magnet application. This may be explained by the drug release profile of the complexes. Additional animal experiments would be needed before the MNP/Ropiv technique can be tested in humans [6]. But if it proved safe, the magnet-directed approach could 


\section{Nanomedicine \& Nanotechnology Open Access}

provide a useful new alternative for regional anesthesia -delivering high concentrations of local anesthetics directly to the desired area, without increasing toxic effects [7].

\section{Conclusion}

It seems that nanotechnology is bound to touch each sphere of life. These are some of the areas where technology will impact the practice of anaesthesiology. Nanotechnology may be considered at the core.

\section{References}

1. Agarwal A (2012) The future of anaesthesiology. Indian J Anaesth 56(6): 524-528.

2. Sinha PM, Valco G, Sharma S, Liu X, Ferrari M (2004) Nanoengineered device for drug delivery application. Nanotechnology 15(10): S585-589.

3. Freitas RA Jr (1998) Exploratory design in medical nanotechnology: A mechanical artificial red cell. Artif
Cells Blood Substit Immobil Biotechnol 26(4): 411430.

4. Freitas RA Jr (2002) The future of nanofabrication and molecular scale devices in nanomedicine. Stud Health Technol Inform 80: 45-59.

5. Mantha VRR, Nair HK, Venkataramanan R, Gao YY, Matyjaszewski K, et al. (2014) Nanoanesthesia: a novel, intravenous approach to ankle block in the rat by magnet-directed concentration of ropivacaineassociated nanoparticles. Anaesth Analg 118(6): 1355-1362.

6. Sedigheh N, Mahmoudvand H, Ali E (2016) Magnetic nanogel polymer of bupivacaine for ankle block in rats. Journal of Microencapsulation 33(7): 656-662.

7. Shen Y, Ji Y, Xu S, Chen DQ, Tu J (2011) Multivesicular liposome formulatons for the sustained delivery of ropivacaine hydrochloride: preparation, characterization and pharmacokinetics. Drug Deliv 18(5): 361-366. 